\title{
COVID-I 9 e a educação: primeira abordagem analítica da situação na Argentina
}

\author{
COVID-I 9 and education: first analytical approach to the situation in \\ Argentina
}

\section{COVID-I 9 y Educación: primer acercamiento analítico a la situación en Argentina}

Gimena Inés Fernández'

https://orcid.org/ 0000-0002-1095-9229

\begin{abstract}
Resumen: El presente artículo presenta una primera lectura del impacto de la pandemia de coronavirus en las prácticas escolares cotidianas. En primer lugar, se describe la situación del sistema educativo argentino a partir de la sanción, en el año 2006, de la Ley Nacional de Educación 26.206 y se analizan las transformaciones del mismo desde entonces. En el contexto actual, se suma como agravante la suspensión de las clases presenciales como consecuencia del COVID-19, que obliga a los actores educativos a tomar decisiones para el sostenimiento de la continuidad pedagógica y configura un escenario particular. Ante esta situación, se presenta un primer acercamiento analítico a la incorporación y uso de las tecnologías digitales en el proceso de enseñanzaaprendizaje. En lo que respecta a lo metodológico, para la elaboración del artículo se recurre al uso de metodologías de investigación propias de las Ciencias Sociales, particularmente: interpretación de documentos oficiales -estadísticas, resoluciones-, entrevistas informales a diferentes actores educativos y sistematización de fuentes secundarias.
\end{abstract}

Palabras-clave: Educación. Pandemia. Tecnologías digitales. Prácticas.

Resumo: Este artigo apresenta uma primeira leitura do impacto da pandemia do coronavírus nas práticas escolares diárias. Em primeiro lugar, descreve-se a situação do sistema educacional argentino após a sanção, em 2006, da Lei Nacional de Educação 26.206 e analisam-se as transformações da mesma desde então. No contexto atual, a suspensão das aulas presenciais em decorrência do COVID-19 acrescenta-se como um agravante, que obriga os atores educacionais a tomarem decisões para manter a continuidade pedagógica e configura um determinado cenário. Diante dessa situação, apresenta-se uma primeira abordagem analítica da incorporação e uso de tecnologias digitais no processo de ensino-aprendizagem. No que diz respeito ao procedimento metodológico, para a elaboração do artigo recorre-se a metodologias de investigação próprias das Ciências Sociais, nomeadamente: interpretação de documentos oficiais -estatísticas, resoluções-, entrevistas informais com diferentes atores educativos e sistematização de fontes secundárias.

Palavras-chave: Educação. Pandemia. Tecnologias digitais. Práticas.

'Professora de Antropologia (FACSO, UNCPBA, Argentina). Doutoranda em Ciências da Educação. (FAHCE, UNLP, Argentina). Bolsista de doutorado em Comissão de Pesquisa Científica da Província de Buenos Aires (Argentina). Membro do Núcleo de Pesquisa Científico-Tecnológica "Pesquisa em Treinamento Inicial e Prática de Ensino” (IFIPRAC.ED, FACSO, UNCPBA, Argentina). Email: gimefernandezI57@gmail.com

Olhar de professor, Ponta Grossa, v. 24, p. I-12, e-16129.0057, 2021.

Disponível em <https://revistas2.uepg.br/index.php/olhardeprofessor> 
Abstract: This article presents a first reading of the impact of the pandemic coronavirus on daily school practices. First, the situation of the Argentine educational system is described as of the sanction, in 2006, of the National Law of Education 26.206 and the transformations of the same since then is analyzed. In the current context, the suspension of face-to-face classes as a consequence of COVID-19 is added as an aggravating factor, which forces educational actors to make decisions to maintain pedagogical continuity and configures a particular scenario. In this situation, a first analytical approach is presented to the incorporation and use of digital technologies in the teaching-learning process. With regard to the methodological, for the preparation of the article the use of research methodologies typical of the Social Sciences is used, particularly: interpretation of official documents -statistics, resolutions-, informal interviews with different educational actors and systematization of secondary sources.

Keywords: Education. Pandemic. Digital technologies. Practices.

\section{Introdução}

Las múltiples transformaciones que inician en la segunda mitad del siglo $X X$ e impactan en las instituciones económicas, políticas, sociales, ideológicas vigentes durante más de dos siglos (Gainza, 2003) configuran un escenario excepcional que se visualiza con mayor precisión en las primeras décadas del siglo XXI. La sociedad actual se encuentra marcada por el cambio de paradigma económico, cuyo objetivo es acrecentar ganancias utilizando la emergencia de las nuevas tecnologías de la comunicación y la información (TIC).

Los cambios estructurales del capitalismo en las últimas décadas, la transformación del Estado nacional, las tensiones entre lo global y lo local, los procesos de desregulación, descentralización y privatización, han incidido en la reconfiguración de las relaciones entre sociedad y educación.

A esta situación social, económica y política en permanente cambio, se suma como agravante el desembarco del Coronavirus (COVID-19) en el mundo, siendo declarado por la Organización Mundial de la Salud (OMS) una pandemia ${ }^{2}$ el II de marzo de 2020. Desde ese momento, la vida cotidiana de los sujetos cambia radicalmente, sobre todo por la recomendación de distanciamiento social y evitar la circulación de personas como única medida efectiva para prevenir el contagio. En Argentina, el I5 de Marzo de 2020, el presidente anunció, entre otras medidas, la suspensión de clases presenciales en los niveles inicial, primario, secundario y terciario a partir del día 16, en primer momento, por catorce días. Esta decisión se plasmó en la Resolución del Ministerio de Educación $\mathrm{N}^{\circ} 108 / 20^{3}$, en donde además se establece la creación del Programa "Seguimos educando", con una propuesta multiplataforma (sitio web, emisiones televisivas y radiales, cuadernillos impresos) que acompañe la enseñanza ante el nuevo contexto y colabore con la continuidad pedagógica en todos los sectores del país, priorizando aquellos más vulnerables (comunidades rurales, en situación de aislamiento, etc.).

\footnotetext{
${ }^{2}$ De acuerdo a los lineamientos de la Organización Mundial de la Salud, para que se declare el estado de pandemia se tienen que cumplir dos criterios: que el brote epidémico afecte a más de un continente y que los casos de cada país ya no sean importados sino provocados por transmisión comunitaria.

${ }^{3}$ Disponible en https://www.argentina.gob.ar/sites/default/files/rs-2020-1702472 I-apn-me.pdf
} 
La aparición de la pandemia de COVID-19 invadió y desbordó la vida cotidiana y las proyecciones a mediano plazo. Pasados más de ochenta días de suspensión de clases presenciales, se puede ver el fuerte impacto en los procesos educativos formales, en el trabajo docente, y una profundización de la desigualdad educativa en los sectores más desfavorecidos. A la metamorfosis que atravesaba y acechaba a la educación en Argentina, se le adiciona el agravante de la pandemia, que la obliga a adoptar nuevas formas. Ni docentes ni estudiantes se encontraban preparados para reconvertirse a un nuevo formato de interacción y producción, y ante esta nueva situación, resulta imperioso tomar decisiones que posibiliten la enseñanza y el aprendizaje en un contexto de no presencialidad.

A partir del análisis preliminar de documentación oficial -relevamientos estadísticos, resoluciones ministeriales, entre otros-, el relevamiento de la experiencia de distintos actores involucrados en el proceso educativo y la sistematización de fuentes secundarias, el objetivo del presente artículo será presentar una primera aproximación al impacto de la pandemia de coronavirus en el funcionamiento del sistema educativo argentino.

Se intentará dar cuenta del proceso de transformación que atravesaba la educación en el país previo a la declaración de pandemia, y cómo ésta obligó a los actores involucrados a tomar decisiones de distinta índole en la emergencia, poniendo en primer lugar el sostenimiento de la continuidad pedagógica y el vínculo con la institución escolar. Esta lectura se complementará con un primer acercamiento analítico a la incorporación de tecnologías digitales en los procesos de enseñanza-aprendizaje, situación que se potenció como consecuencia de la no presencialidad escolar.

\section{El sistema educativo argentino desde la normativa}

En Argentina, la influencia de organismos internacionales contribuyó a la conformación de una agenda educativa que contemple la reforma de los marcos regulatorios como así también una redefinición de sentidos, discursos y/o prácticas asociadas a la educación en nuestro país.

En el año 2003, comenzaron las reformas en las normativas destinadas a atender a las problemáticas del sistema educativo, consecuencia de la fragmentación y desfinanciamiento de la educación durante la década del '90. Finnegan y Pagano (2007) consideran fundamental la sanción de tres leyes que allanaron el camino para la sanción de la Ley Nacional de Educación Nº 26206 en el año 2006: Ley de Garantía de la duración del Ciclo Lectivo N²5864/04; Ley de Educación Técnico Profesional N ${ }^{\circ}$ 26058/05; y la Ley de Financiamiento Educativo N ${ }^{\circ} 26075 / 05$.

En este sentido, en el año 2006 se sanciona la Ley de Educación Nacional N²6.206 (LEN). Se propone como concepto central que articula la propuesta la inclusión educativa. En este sentido, tanto el Ministerio de Educación como el Consejo Federal de Educación, fijarán y desarrollarán políticas de 
COVID-19 e a educação: primeira abordagem analítica da situação na Argentina

promoción de la igualdad educativa, destinadas a enfrentar situaciones de injusticia, marginación y discriminación. A partir de ello, se intenta garantizar el acceso y la permanencia de los alumnos en todos los ciclos y modalidades. Al mismo tiempo, se produce la ampliación de la obligatoriedad, desde el nivel inicial hasta la finalización de la escuela secundaria.

La educación debe enfrentarse al desafío de consolidar la expansión, pero esto está ligado a la transformación de la identidad, procesos internos y formas de organización del nivel medio. Sin esta reforma integral, el objetivo de garantizar una educación inclusiva, equitativa y de calidad, como se promueve desde Naciones Unidas en la Agenda 2030 para el Desarrollo Sostenible ${ }^{4}$, y otros organismos internacionales con injerencia en la política educativa regional y nacional, se verá seriamente comprometido; será difícil masificar la escolarización hasta el nivel secundario, de manera sostenible y con sentido para los jóvenes (Pedró, 2017). En pos del trabajo para el logro de objetivos y metas planteados a nivel macro, el Ministerio de Educación trabaja de forma mancomunada con distintas organizaciones, como la Comisión Económica para América Latina y el Caribe (CEPAL), la Organización de Estados Iberoamericanos para la Educación, la Ciencia y la Cultura (OEI), o el Instituto Internacional de Planeamiento de la Educación (IIPE-UNESCO), por citar algunos ejemplos

Pedró (op.cit.) sostiene que sin una modificación en los contenidos curriculares, las estrategias de enseñanza -entre otros aspectos, que se traduzca en más y mejores competencias para los estudiantes, será difícil que los jóvenes puedan aprovechar las oportunidades que ofrecen la sociedad interconectada y la economía del conocimiento.

Desde el discurso de los actores que toman decisiones respecto al funcionamiento del sistema educativo, se reconoce la necesidad de mejorar la calidad de la enseñanza, adaptándola a los requerimientos de una sociedad digital e interconectada, por lo que se propone la incorporación de las TIC al proceso de enseñanza-aprendizaje. Sin embargo, no se evidencia un análisis crítico y reflexivo en torno a la potencialidad que ofrece el uso de las tecnologías digitales en el aula y su impacto en los aprendizajes, como tampoco la formación docente para ese fin y la modificación, adaptación y/o actualización de los contenidos curriculares de acuerdo a las demandas de la sociedad actual.

\section{El desafío de la "continuidad pedagógica" en pandemia}

El Ministerio de Educación de la Nación, junto con representantes de las jurisdicciones provinciales y especialistas en investigación educativa, conformó un grupo de trabajo con el propósito de brindar precisiones sobre el rumbo del sistema educativo argentino en este escenario excepcional. Desde el posicionamiento de la política educativa, se sostuvo que asegurar la continuidad pedagógica

\footnotetext{
${ }^{4}$ Ver Objetivos de Desarrollo Sostenible - Naciones Unidas: https://www.un.org/sustainabledevelopment/es/
} 
implica, por un lado, considerar el impacto de la discontinuidad del proceso de enseñanza-aprendizaje en los sujetos involucrados en el acto educativo; y por otro, "ofrecer propuestas de enseñanza que permitan aprendizajes valiosos ante las distintas situaciones o contextos que así lo demanden" (Dirección General de Cultura y Educación de la Provincia de Buenos Aires, 2020, p. 4).

En este sentido, el primer punto sobre el que poner énfasis, que ocupó las primeras semanas de aislamiento y coincidió con el inicio del ciclo lectivo 2020, fue la comunicación con las y los estudiantes. Identificarlos, generar algún tipo de conexión con ellos, conocer en qué situación socioeconómica se encontraban y la posibilidades para el sostenimiento de la educación de forma no presencial. En aquellos casos en los que se contaba con conectividad a internet, regular y constante, la posibilidad del dictado de clases virtuales fue la solución.

Para los sectores con dificultades de conectividad -sea por causas socioeconómicas, ubicación geográfica, etc.-, se organizó la distribución de cuadernillos de trabajo, programas de radio y televisión, diseñados por especialistas del Ministerio de Educación de Nación, a sabiendas de que el proceso de enseñanza-aprendizaje sería sustancialmente diferente del que puedan llevar adelante aquellos quienes tengan acceso al dictado de clases con mediación de tecnologías digitales y con la posibilidad de contar con el intercambio con el docente.

De acuerdo con el relevamiento realizado por Fundación VOZ (2020), si se considera la totalidad de la extensión geográfica de Argentina, se pueden identificar situaciones muy heterogéneas. El rastreo y la identificación de la población estudiantil pusieron en evidencia las desigualdades -sociales y económicas- que atraviesa nuestro país, con cientos de miles de estudiantes sin posibilidad de conexión y/o dispositivos que les permitan mantener el contacto.

En definitiva, los esfuerzos cotidianos de estudiantes, docentes y directivos para transformar la educación, desde el punto de vista curricular y también desde las estrategias metodológicas, se vieron interrumpidos a partir de la suspensión del dictado de clases presenciales el 16 de Marzo de 2020, como consecuencia del avance de la pandemia de COVID-19.

Ángel Díaz Barriga en la publicación del Instituto de Investigaciones sobre la Universidad y la Educación-UNAM (2020), reflexiona sobre lo acontecido en México, y sostiene que "la preocupación que ha orientado todas estas decisiones es <salvar> el año escolar, no necesariamente analizar las opciones de aprendizaje que esta circunstancia ofrece a los alumnos" (p. 24). La decisión de hacer énfasis en la continuidad pedagógica, la comunicación y el establecimiento de vínculos en una primera etapa del aislamiento, sumado al desconcierto que docentes y directivos manifestaron en entrevistas informales permite establecer algún tipo de relación entre la experiencia mexicana y la argentina. 
COVID-19 e a educação: primeira abordagem analítica da situação na Argentina

En este sentido, el Mg. Becerra, en entrevista con Revista Marfil ${ }^{5}$, hace referencia a un desplazamiento del eje de reforma del sistema sobre el que se trabajaba desde el Ministerio de Educación nacional, con énfasis en la calidad y la mejora del aprendizaje, y se dio prioridad, en contexto de pandemia, a la continuidad pedagógica, al sostenimiento de los vínculos interpersonales, con el objetivo de garantizar el derecho social a la educación.

En una misma línea, Rivas (2020) considera que el escenario de pandemia generó la ruptura de todos los esquemas de la escolarización como se la conocía: no hay presencia ni encuentro en un espacio físico, no hay horarios ni rutinas encuadradas, se desarmó el currículum y desapareció la motivación basada en el deber y el cumplimiento de la norma. Es por eso que se volvió completamente necesario tomar decisiones para establecer un vínculo pedagógico en un contexto no presencial, o en el mejor de los casos, en un entorno digital.

La cultura digital y las nuevas tecnologías ya son parte de la cotidianeidad, pero eso no implica que deban reemplazar la cultura escolar en su totalidad, ni que mejoren la educación de forma automática (Sewlyn, 2016). Sin embargo, para brindar la sensación de continuidad y establecer secuencias de aprendizaje, en aquellos sectores en los que se cuenta con conectividad a internet, la incorporación de plataformas de enseñanza virtual (PEV) resulta una herramienta sumamente útil. Éstas han sido definidas por José Sánchez (2009), como "un amplio rango de aplicaciones informáticas instaladas en un servidor cuya función es la de facilitar al profesorado la creación, administración, gestión y distribución de cursos a través de Internet" (p. 218). Son aplicaciones que permiten dar una estructura a la/las actividades propuestas, visualizar el proceso de forma continua, fomentar el intercambio y la retroalimentación entre docentes y estudiantes, generando una red de acompañamiento pedagógico.

En el caso argentino, la utilización de PEV quedó librada a las decisiones jurisdiccionales. En este sentido, provincias del interior desarrollaron nuevas plataformas o actualizaron/adaptaron las que ya tenían en funcionamiento. La implementación no sólo quedó en manos de las jurisdicciones y/o autoridades educativas provinciales, sino también en supervisores, jefes $y / 0$ inspectores regionales, como así también en los equipos directivos de cada institución que, frente a la variedad de oferta de plataformas de enseñanza disponibles en la web, optaban por una u otra sin hacer explícito -en los casos relevados- los criterios de selección de las mismas. También se pudo relevar en entrevistas con docentes de distintos niveles que, en ocasiones, recurrieron al uso de redes sociales como espacio de intercambio de materiales y/o actividades o como complemento del uso de una PEV:

"Como trabajo en primer grado, con nenes chiquitos, mi contacto siempre es con los papás... Primero armé un grupo de WhatsApp, pero ahí daba para todo tipo de mensajes, información de cualquier cosa, videos... Así que decidí hacer listas de difusión por

\footnotetext{
${ }^{5}$ Disponible en https://revistamarfil.com/2020/06/2I/educacion-en-tiempos-de-pandemia/
} 
WhatsApp también, con lecturas y consignas, y luego que cada padre me envíe la producción (...)" (C., docente I grado nivel primario, abril 2020).

“Inicialmente recurrí al grupo de WhatsApp, en el que podía generar un ida y vuelta con mis estudiantes Después empecé a notar que no tenía un límite, trabajaba 24/7, entonces empecé a buscar otras estrategias y elegí crearme un Google Classroom (...)" (MP., docente $\mathrm{I}^{\circ}$ año nivel secundario, mayo 2020).

“El equipo de gestión de la escuela nos pidió que armemos grupos privados de Facebook, por materia, con presencia de estudiantes o un familiar directo en caso de que no tengan usuario por la edad... Ahí subimos los materiales y la idea es generar diálogo en las publicaciones, aunque los chicos prefieren mandarte mensaje privado y no exponerse (...)" (J, docente $3^{\circ}$ año nivel secundario, mayo 2020).

"El instituto usaba Google Classroom como opcional y ahora lo hizo obligatorio... Cada profesor creó un aula con sus estudiantes y funciona casi como un campus virtual... Eso se complementa con un grupo de WhatsApp en donde están los preceptores y los estudiantes. Ellos son los encargados de notificarnos si alguno de los chicos tiene una dificultad para ingresar a Classroom. (...)" (M., docente $6^{\circ}$ año nivel secundario, junio 2020).

Independientemente de las realidades diversas o el nivel educativo en que los docentes se desempeñan, el esfuerzo por sostener el vínculo con los estudiantes, o sus familias como interlocutores, es un punto en común.

Una vez restablecido el contacto, llegó el momento de tomar decisiones respecto a qué y cómo enseñar, hacer una selección de contenidos curriculares y estrategias metodológicas para la enseñanza en entornos digitales. Según Rivas (op.cit.) este fue el momento "para pensar y hacer buenas propuestas de aprendizaje, que tengan en cuenta los saberes previos de los estudiantes y sus condiciones actuales" (p. 6). A este escenario, de planificación y acción de forma paralela, y frente a la incertidumbre de la situación sanitaria, se le debe sumar la complejidad que supone la enseñanza de las disciplinas específicas. Cabe destacar que, la formación docente inicial en Argentina tiene una tradición enciclopedista, por lo que esta pandemia obligó a revisar los supuestos y las bases epistemológicas con el objetivo de delinear estrategias de enseñanza que posibiliten aprendizaje significativo en los estudiantes.

Para esto, es necesario el diálogo con los colegas que fomente la interdisciplina, el aprendizaje colaborativo entre pares, la experimentación a partir de situaciones cotidianas, y todas aquellas estrategias y herramientas que generen en el estudiante el deseo de aprender. Una vez establecida una secuencia didáctica, basada en una economía de contenidos, a través de temas y problemas centrales de la disciplina a enseñar, es necesario re-establecer una rutina de trabajo, por lo tanto, las estrategias de abordaje resultan fundamentales.

En relación a los vínculos y al aspecto socioemocional que los y las docentes debieron atender en la no presencialidad, se puede encontrar en su relato las múltiples estrategias desplegadas para relevar la situación de los y las estudiantes en el contexto de aislamiento. En aquellos sectores donde la conectividad estaba presente, se instrumentaron estrategias vinculadas con recursos web: encuestas

Olhar de professor, Ponta Grossa, v. 24, p. I-12, e-16129.057, 2021.

Disponível em <https://revistas2.uepg.br/index.php/olhardeprofessor> 
COVID-19 e a educação: primeira abordagem analítica da situação na Argentina

elaboradas con herramientas digitales -como Formularios de Google-, reuniones virtuales en plataformas de videoconferencia para dialogar sobre las emociones y sentimientos de los y las jóvenes en el período de aislamiento, entre otras. Mientras que en aquellos sectores más desfavorecidos, con escasa o nula conectividad a internet, el vínculo se sostuvo a través de los equipos directivos, orientadores sociales y personal auxiliar que obtuvo habilitación para concurrir al establecimiento educativo con el propósito de realizar actividades administrativas. De entrevistas con docentes, se rescatan los siguientes fragmentos que apuntan a la experiencia de establecimiento y/o sostenimiento de vínculos sociales, afectivos y comunitarios en el período de ASPO:

“Un mes después del inicio del aislamiento ya habíamos logrado organizar una lógica de dictado de clases, pero se me hizo necesario organizar una reunión por videollamada, mediante Zoom, para conversar sobre cómo se sentían ellos, cómo estaban atravesando esta situación... Normalmente yo soy mucho más que su profesora, soy una compañera, y la suspensión de clases también suspendió la vinculación con el grupo desde otro lugar, desde la cercanía, el consejo (...)" (E., docente de 4to, 5to y 6to año nivel secundario, septiembre 2020).

"Desde el inicio del aislamiento, las clases estaban organizadas mediante un aula de Google Classroom, sin embargo, después de unas 3 semanas, viendo que la situación se prolongaba, se me hizo necesario instrumentar una encuesta anónima, que cree con Formularios de Google, para conocer cómo se sentían mis estudiantes, qué emociones o sentimientos los habían invadido desde el inicio del ASPO, a quién recurrían cuando necesitaban ayuda, cuáles de las tareas los motivaba más... No sé, indagué en varias cuestiones... Eso me permitió acercarme más a ellos, conocer algunas de sus preferencias, sus saberes en relación a la tecnología también... Ellos lo valoraron como positivo y yo también, porque me permitió planificar las clases de manera diferente, contemplando la valoración que los estudiantes hacían de mis propuestas (...)" (G., docente de 5 to año de nivel secundario, mayo 2020).

“Antes de la suspensión de clases estaba muy entusiasmada porque tenía referencias muy buenas del grupo de estudiantes... No alcancé a tener ningún encuentro presencial con ellos... Eso me desanimó un poco, porque no pude tener ningún tipo de acercamiento ni establecer un vínculo con ellos... Por eso se me ocurrió organizar una mateada virtual, por videollamada, para conocer sus caras y también para poder establecer un vínculo desde otro lado que no sea lo curricular... Desde entonces organizamos este tipo de encuentros regularmente, donde la idea es encontrarnos, no hablar sobre las tareas o contenidos (...)" (G., docente de 6to año de nivel secundario, septiembre 2020).

"Durante la primera etapa del aislamiento tuvimos relación con muchos de nuestros estudiantes... Pero teníamos tantos que no... Realmente era preocupante, y sigue siendo hoy... Cuando nos dieron el permiso para ingresar a la escuela, el equipo directivo y las orientadoras sociales empezamos a contactar a las familias... Algunos padres vinieron con los chicos a la escuela para que charlemos sobre lo que estaban viviendo... A otros los fuimos a visitar desde el equipo... Nos organizamos para ver si teníamos alguna oportunidad de revincular a esos estudiantes con el colegio..." (M., secretaria de establecimiento educativo de nivel secundario, agosto 2020).

Tal como se puede apreciar en el relato de los docentes, al dictado de clases y trabajo con los contenidos curriculares, se sumó una instancia de vinculación y comunicación con los y las estudiantes 
desde el punto de vista emocional, social y afectivo. Este tipo de actividades regularmente se realizan en el salón de clases, en la construcción del grupo-clase, de la comunidad de enseñanza-aprendizaje, es algo inherente a la relación docente-alumnos, pero la no presencialidad complejizó la vinculación y obligó al diseño de estrategias -mediadas o no por tecnologías digitales- para establecer relaciones sociales.

\section{Consideraciones finales}

Históricamente, el sistema educativo en Argentina ha sido objeto de cuestionamientos y múltiples reformas. Sin embargo, a partir de la sanción de la LEN en 2006, el Estado se asumió como el responsable de garantizar el acceso a la educación y al conocimiento, entendidos como derechos personales y sociales. Para ello, desarrolló e implementó múltiples políticas y programas, en pos de la inclusión educativa y la mejora de la calidad de la enseñanza y los aprendizajes. Sin embargo, en la práctica cotidiana, la implementación efectiva de las mismas se llevó a cabo de manera dispar, sin dar respuesta a las demandas formativas de la sociedad actual ni generar instancias de apropiación significativa de las tecnologías digitales en el ámbito educativo. Para citar algunos ejemplos, se puede hacer referencia a Conectar Igualdad y Aprender Conectados, dos de las más recientes políticas de alfabetización digital con énfasis en la inclusión y la calidad, respectivamente, en línea con los objetivos para la educación de los gobiernos que las diseñaron (Benitez Larghi y Welschinger, 2020).

A la situación ya descripta, se sumó como agravante la pandemia de Coronavirus, que ocasionó la suspensión de las clases presenciales, una "migración masiva” a la enseñanza mediada por tecnologías, en plataformas virtuales en el mejor de los casos y una profundización de la desigualdad en aquellos sectores más vulnerables. Jóvenes estudiantes de escuelas empleadas en barrios o áreas de periferia de las ciudades, con múltiples carencias materiales y afectivas, que regularmente cursan sus estudios en establecimientos educativos con problemas edilicios, falta de personal docente y de equipamiento, como los describe Sassera (2018), son aquellos que el sistema no ha logrado incluir con eficiencia desde la implementación de la Ley Nacional de Educación, y que la pandemia agudizó la desigualdad educativa.

En otro plano de análisis, y como consecuencia de los múltiples formatos que se adoptaron para sostener la continuidad pedagógica en el marco de la pandemia, resulta necesario revalorizar la labor de los actores educativos implicados en la cotidianeidad del proceso de enseñanza-aprendizaje. De un día a otro se encontraron frente a la suspensión de la presencialidad, lo que significó salir a buscar a los y las estudiantes, realizar un diagnóstico real de la situación social y económica de los jóvenes y sus familias y diseñar dispositivos que les permitan mantenerse conectados con la escuela y la enseñanza. Tomar decisiones didácticas en la emergencia, migrar a espacios digitales, revisar diseños

Olhar de professor, Ponta Grossa, v. 24, p. I-12, e-16129.057, 2021.

Disponível em <https://revistas2.uepg.br/index.php/olhardeprofessor> 
curriculares y planificaciones, explorar herramientas digitales, repensar expectativas de logro y objetivos para un período que parece nunca tener fin.

En relación a este último planteo, a la búsqueda incesante de estrategias para trabajar con los y las estudiantes, es importante resaltar que se habilitaron numerosos ciclos de conferencias, ateneos y/o webinars ${ }^{6}$, en donde participaron miembros de equipos de trabajo del Ministerio de Educación de la Nación, como así también referentes del ámbito de la didáctica y la investigación educativa de Argentina y la región, como Rebeca Anijovich, Inés Dussel, Mariana Maggio, Laura Mares, Marilina Lipsman, Carina Lion, Manuel Becerra, Cecilia Sagol, entre muchos otros. En estos ámbitos, se hizo visible consenso respecto a la necesaria revisión de los contenidos curriculares y su pertinencia, hacer una selección en cantidad y calidad, priorizando la producción de sentido, la motivación, el deseo de saber. En este sentido, se puede pensar en una capitalización de las experiencias de enseñanza y aprendizaje, mediadas o no por tecnologías digitales, que se desarrollaron como consecuencia de la pandemia. Tomarlas como un punto de partida para la reinvención y/o reformulación del sistema educativo en el país que se venía trabajando previo a la emergencia sanitaria.

Las consecuencias de la pandemia en la educación aún no pueden dimensionarse. Sin fecha de retorno al espacio físico de la escuela, al aula, a la clase presencial, al encuentro, solamente hay interrogantes: ¿cuáles de las decisiones, estrategias, herramientas adoptadas durante este período permanecerán en las prácticas educativas? ¿será, en el futuro próximo, el modelo híbrido de educación el que nos permita continuar el proceso de enseñanza-aprendizaje? y por último, pero no por eso menos importante, sino más bien todo lo contrario ¿qué pasará con los miles de estudiantes con los que se perdió contacto debido a la falta de conectividad y dispositivos tecnológicos?

\section{Referências}

BENITEZ LARGHI, S. y WELSCHINGER, N. (2020) "Once puntos para una futura política de inclusión digital”. Publicado en Revista Anfibia: http://revistaanfibia.com/ensayo/once-desafios-parael-futuro-del-conectar-igualdad/

\section{DIRECCIÓN GENERAL DE CULTURA Y EDUCACIÓN. Propuestas para la continuidad} pedagógica en la Educación Secundaria. Ciclo Superior. Buenos Aires: Autor, 2020. Disponible en: http://abc.gob.ar/secundaria/sites/default/files/documentos/ciclosuperior.pdf

FINNEGAN, F. y PAGANO, A. El derecho a la educación en Argentina. Buenos Aires: Fundación Laboratorio de Políticas Públicas, 2007. Disponible en: http://www.derechoshumanos.unlp.edu.ar/assets/files/documentos/el-derecho-a-la-educacion-enargentina.pdf

\footnotetext{
${ }^{6}$ Convocatorias y eventos online, libres y gratuitos, organizados por organismos internacionales -UNICEF, OEI-, universidades nacionales y otras instituciones vinculadas al ámbito educativo.
}

Olhar de professor, Ponta Grossa, v. 24, p. I-12, e-16/29.057, 2021.

Disponível em <https://revistas2.uepg.br/index.php/olhardeprofessor> 
GAINZA, C. "Sociedad, Estado y Tecnología: ¿qué pasa hoy en nuestras sociedades". En Revista de Sociología de la Facultad de Ciencias Sociales. $N^{\circ}$ 17. Universidad de Chile, 2003. Disponible en: https://revistadesociologia.uchile.cl/index.php/RDS/article/view/27794

FUNDACIÓN VOZ. “¿Cómo continuamos después de la cuarentena?”. En Volver a las escuelas. Ciclo de consultas intersectoriales. Junio 2020. Disponible en: https://panorama.oei.org.ar/volver-a-clases-como-continuamos-despues-de-la-cuarentena/

INSTITUTO DE INVESTIGACIONES SOBRE LA UNIVERSIDAD Y LA EDUCACIÓN. Educación y pandemia. Una visión académica. México: UNAM, 2020. Disponible en:

http://www.iisue.unam.mx/nosotros/covid/educacion-y-pandemia

Ley de Garantía de la duración del Ciclo Lectivo № 25864/04. Disponible en: https://www.argentina.gob.ar/normativa/nacional/ley-25864-91819

Ley de Educación Técnico Profesional N²6058/05. Disponible en: https://www.argentina.gob.ar/normativa/nacional/ley-26058-109525

Ley de Financiamiento Educativo $N^{\circ} 26075 / 05$. Disponible en:

https://www.argentina.gob.ar/normativa/nacional/ley-26075-I I 2976

Ley de Educación Nacional $N^{\circ}$ 26.206/06. Disponible en: https://www.argentina.gob.ar/sites/default/files/ley-de-educ-nac-58ac89392ea4c.pdf

PEDRÓ, F. Tecnologías para la transformación de la educación. España: Fundación Santillana, 2017. Disponible en:

http://www.fundacionsantillana.com/PDFs/Tecnologias\%20para\%20la\%20transformacion\%20de\%20la\% 20educacion.pdf

SÁNCHEZ RODRÍGUEZ, J. "Plataformas de enseñanza virtual para entornos educativos". En PixelBit. Revista de Medios y Educación. №34. España: Universidad de Sevilla, 2009. Disponible en: https://www.redalyc.org/pdf/368/368I20360I5.pdf

SASSERA, J. "Segmentación educativa y políticas educativas es espacios locales de Buenos Aires". En BUENAVENTURA RODRÍGUEZ, M. [et al.]. Nuevos desafíos en educación. Una mirada interdisciplinaria. Buenos Aires: FLACSO Argentina, 2018. Disponible en:

https://www.flacso.org.ar/publicaciones/nuevos-desafios-en-educacion-una-mirada-interdisciplinaria/

SEWLYN, N. "Profesores y tecnología: repensar la digitalización de la labor docente". En Boletín de la Institución Libre de Enseñanza. N ${ }^{\circ}$ I04. 2016. Disponible en:

https://www.researchgate.net/publication/326 |24373_Profesores_y_tecnologia_repensar_la_digitaliz acion_de_la_labor_docente

RIVAS, A. Pedagogía de la excepción: ¿cómo educar en la pandemia?. Documento de trabajo. Buenos Aires: Universidad de San Andrés, 2020. Disponible en:

https://www.udesa.edu.ar/sites/default/files/rivas-educar_en_tiempos_de_pandemia.pdf

Olhar de professor, Ponta Grossa, v. 24, p. I-12, e-16129.057, 2021.

Disponível em <https://revistas2.uepg.br/index.php/olhardeprofessor> 
Recebido em: 15 de junho de 2020.

Versão corrigida recebida em: 10 de novembro de 2020 .

Aceito em: 10 de novembro de 2020.

Publicado online em: 04 de junho de 2021.

(c) (i) 